\title{
In vitro activity of fluralaner and commonly used acaricides against Dermanyssus gallinae isolates from Europe and Brazil
}

Emmanuel Thomas ${ }^{1 *}$, Hartmut Zoller ${ }^{1}$, Gabriele Liebisch², Luis Francisco Angeli Alves ${ }^{3}$, Luis Vettorato ${ }^{4}$, Rafael M. Chiummo ${ }^{4}$ and Annie Sigognault-Flochlay ${ }^{5}$

\begin{abstract}
Background: The poultry red mite Dermanyssus gallinae negatively impacts bird welfare and health, and interferes with egg production and quality, while emerging acaricide resistance limits control options. Fluralaner, a novel miticide for administration in drinking water, is approved for control of D. gallinae infestations. Mite sensitivity testing is relevant to gauge field isolate susceptibility to available treatments.

Methods: Thirteen D. gallinae isolates collected during 2014 through 2016 from farms in Germany, France, Spain and Brazil, and a 2001 laboratory-maintained isolate were used for acaricide contact sensitivity testing. Tested compounds were cypermethrin, deltamethrin, phoxim, propoxur, and the recently available acaricides, spinosad and fluralaner. In each study, at least one isolate was exposed to increasing concentrations of at least one acaricide. In one study, additional testing determined the sensitivity of the 2001 isolate to fluralaner using a mite-feeding test, and of fluralaner, phoxim and spinosad using an immersion test. At least two replicates were used for each dilution. Vehicle and untreated controls were also included.

Results: Based on 90\% mortality ( $\left(\mathrm{C}_{90}\right)$ values, the laboratory isolate was susceptible to fluralaner (15.6-62.5 parts per million, ppm), phoxim (<500 ppm), propoxur (<125 ppm), and deltamethrin (500-1000 ppm). All field isolates remained sensitive to fluralaner concentrations $\leq 125 \mathrm{ppm}$. Spinosad $\mathrm{LC}_{90}$ values for laboratory and field isolates ranged between 2000-4000 ppm. For phoxim, relative to the laboratory isolate, there was reduced sensitivity of two German isolates ( $L C_{90}$ up to 4000 ppm) and two French isolates (> 4000 ppm). An isolate from Spain demonstrated reduced sensitivity to phoxim, propoxur and deltamethrin; an isolate from Brazil showed reduced sensitivity to propoxur and cypermethrin. Mite $L_{90}$ when exposed to fluralaner by blood feeding was $<0.1 \mathrm{ppm}$.

Conclusions: Contact sensitivity testing indicated apparent resistance to at least one of phoxim, deltamethrin, cypermethrin and propoxur in 13 field isolates from Europe and Brazil. All isolates were highly susceptible to fluralaner. Fluralaner was approximately 1000 times more active by feeding than by contact. Fluralaner's distinct mode of action and efficacy against isolates largely refractory to those acaricides, makes it a promising option for the control of D. gallinae infestations of poultry.
\end{abstract}

Keywords: Contact toxicity, Cypermethrin, Deltamethrin, Dermanyssus gallinae, Feeding, Fluralaner, Immersion, Phoxim, Propoxur, Spinosad

\footnotetext{
* Correspondence: emmanuel.thomas@msd.de

'MSD Animal Health Innovation GmbH, Zur Propstei, 55270 Schwabenheim,

Germany

Full list of author information is available at the end of the article
}

(C) The Author(s). 2018 Open Access This article is distributed under the terms of the Creative Commons Attribution 4.0 International License (http://creativecommons.org/licenses/by/4.0/), which permits unrestricted use, distribution, and reproduction in any medium, provided you give appropriate credit to the original author(s) and the source, provide a link to the Creative Commons license, and indicate if changes were made. The Creative Commons Public Domain Dedication waiver (http://creativecommons.org/publicdomain/zero/1.0/) applies to the data made available in this article, unless otherwise stated. 


\section{Background}

The poultry red mite Dermanyssus gallinae (De Geer, 1778) is an important ectoparasite of poultry that negatively impacts poultry health and welfare, reduces egg production and egg quality, and is a potential pathogen vector [1-8]. Additionally, mite infestations of poultry workers and of city dwellers with no connections to poultry have been reported as a significant public health concern [9-11]. An ubiquitous hematophagous ectoparasite, D. gallinae seeks its hosts at night, taking blood meals that typically last for less than two hours, after which mites retreat into cracks and crevices of nest boxes or other suitable sites within the wider surroundings of a poultry housing unit [6, 7, 12-14]. At temperatures of $10-37{ }^{\circ} \mathrm{C}$ (with $25{ }^{\circ} \mathrm{C}$ considered optimum), and relative humidity ranging from 70 to $90 \%$, the life-cycle of D. gallinae may be as short as 7 days [8, 15-17]. Such conditions may result in a weekly doubling of the mite population in poultry houses, with up to 500,000 mites potentially infesting one hen in severe cases $[5,7,14]$.

Until recently, the control of the poultry red mite has been largely dependent upon the use of sprayed acaricides, such as organophosphates, carbamates, macrocyclic lactones, pyrethroids, and spinosyns $[8,14]$. However, increasingly restrictive legislation has presented a practical constraint for producers attempting to control this parasite, in some cases leading to illegal or off-label use of banned pesticides, such as carbaryl and fipronil, with risk of food-chain residues [2, 4, 18, 19]. Moreover, repeated acaricide applications, particularly if at incorrect dosages, has led to the emergence of resistance in poultry red mite populations $[4,8]$. Resistance to approved and non-approved acaricides, including alpha-cypermethrin, bifenthrin, carbamates, carbaryl, cypermethrin, deltamethrin, dichlorodiphenylytrichloroethane (DDT), dichlorvos, fenitrothion, fipronil, flumethrin, flurathiocarb, malathion, permethrin, phenothrin, tetramethrin and trichlorfon, has been reported from many countries, including Czechoslovakia, France, Italy, Korea, Montenegro, Poland, Serbia and Sweden [3, 18, 20-24]. Along with a move to housing systems which favour mite proliferation, an outcome of the European Union (EU) directive banning the use of traditional cages since 2012, the emergence of resistance complicates parasite control measures, potentially leading to higher treatment frequencies and increased risk of egg residues [4, 16]. As a result the control of $D$. gallinae by effective and safe treatments has been a largely unmet need for nearly two decades [4].

During 2017, the isoxazoline compound fluralaner was approved for the control of D. gallinae on EU poultry operations [25]. This approval was the outcome of a development programme in which fluralaner, administered in drinking water at a dose rate of $0.5 \mathrm{mg} / \mathrm{kg}$ on two occasions with a 7-day interval, was shown to be safe and to have a zero day egg withdrawal period following treatment. Mite killing has been shown to begin within four hours following an initial fluralaner administration, and on commercial farms in France, Germany and Spain efficacy reached $97.8-100 \%$ as soon as two days following the second administration [26, 27]. Fluralaner selectively targets distinct binding sites on insect and arachnid ligand-gated chloride channels in the nervous system, leading to parasite paralysis and death. This mode of action is different from that of any other poultry acaricide, and cross-resistance between fluralaner and traditional chemicals which act by contact is unlikely [28].

To determine the sensitivity of field isolates of $D$. gallinae to fluralaner, and to assess the sensitivity of these isolates to commonly used mite control acaricides, six in vitro laboratory studies were initiated. Mite isolates were collected for testing from commercial poultry operations in Europe and Brazil. Comparator active ingredients were spinosad, phoxim, propoxur, and the pyrethroids cypermethrin and deltamethrin.

\section{Methods \\ Study design}

Across six separate studies, 12 field isolates were freshly collected between May 2014 and July 2015 from commercial layer, breeder, and pullet farms located in Germany, France and Spain, and in September 2016 one isolate was collected from a layer farm in Brazil. No chemical acaricidal treatment had been used in the sampled houses for at least two months prior to mite collection. In Studies 1 through 5, mites were brushed from typical refuge areas into plastic containers that contained filter paper to absorb humidity, and that had a lid-hole covered with air-permeable paper to preclude mite escape (Fig. 1). In Study 6, mites were collected in traps (Avivet, Lunteren, Netherlands) that had been placed in laying houses for 48 hours prior to collection. Following mite collection containers were taken directly or shipped overnight to the testing laboratory. The laboratory isolate used in Studies 1 and 5 was originally collected from a German commercial layer farm in 2001, and had been maintained at MSD Animal Health Innovation (Schwabenheim, Germany).

Fluralaner was sourced from MSD Animal Health Innovation; commercially available phoxim, deltamethrin, and propoxur were sourced from Sigma-Aldrich Chemicals (Allentown, USA); spinosad from APIN Chemicals (Abingdon, UK), and cypermethrin from Tagros Chemicals (Chennai, India). All compounds tested were of technical grade greater than $95 \%$.

The in vitro contact sensitivity of each of the $13 D$. gallinae field isolates and a laboratory isolate was tested in at least one of three separate laboratories: MSD Animal Health Innovation $\mathrm{GmbH}$, Schwabenheim, Germany (Study 1; field isolate 1); ZeckLab, Burgwedel, Germany 


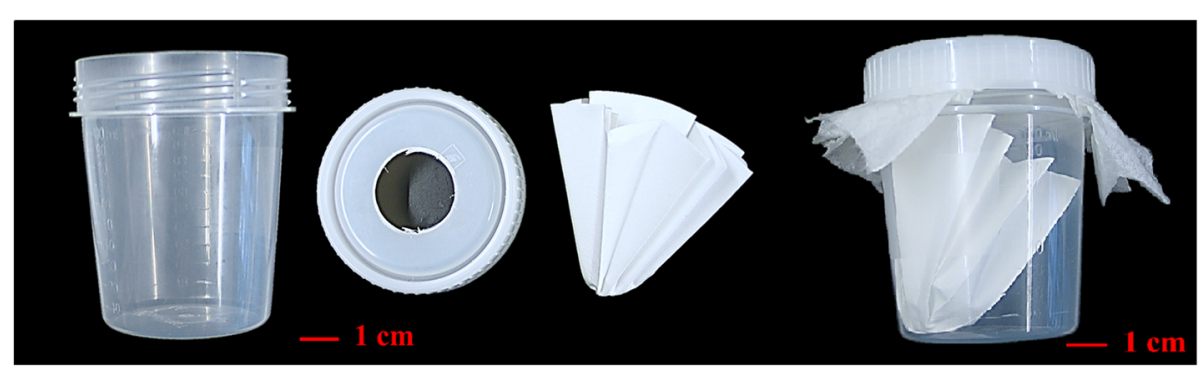

Fig. 1 Plastic container used for collection of mites from poultry houses

(Studies 2 to 5; field isolates 1-12), and the Agricultural Biotechnology Laboratory, State University of Western Paraná, Cascavel, PR, Brazil (Study 6, field isolate 13) (Table 1). Study 1 also included a feeding test and an immersion contact test. In all studies, after removal from test media and incubation, mite mortality (MM) was assessed using a binocular microscope to count all mites, living and dead. If present, moribund mites were counted as dead. Nymphal and adult stages were not differentiated.

\section{Sensitivity testing \\ Contact sensitivity}

Contact sensitivity assays using impregnated filter paper were modifications of the Larval Package Test recommended by the Food and Agriculture Organization (FAO) for resistance testing of tick larvae [29]. Two replicate sets of filter paper were assessed for each control and each concentration of active. In Studies 1 and 6, actives were dissolved in $2.5 \mathrm{ml}$ dimethyl sulfoxide to obtain a stock solution, $2 \mathrm{ml}$ of which were mixed with 38 $\mathrm{ml}$ of deionized water to obtain a test concentration of $1000 \mathrm{ppm}$. Five $\mathrm{ml}$ of test solution or solvent control were pipetted onto a filter paper (185 mm diameter) and

Table 1 Study design summary

\begin{tabular}{|c|c|c|}
\hline Study & Assay & Concentrations tested (ppm) \\
\hline \multirow[t]{3}{*}{1} & Contact & $\begin{array}{l}\text { Fluralaner, phoxim, spinosad: } \\
\text { 3.9-4000 }\end{array}$ \\
\hline & Immersion & $\begin{array}{l}\text { Phoxim, spinosad: } 2.0-4000 \text {; } \\
\text { fluralaner: } 0.3-4000\end{array}$ \\
\hline & Feeding & Fluralaner $10^{-7}-10$ \\
\hline 2 & Contact & Deltamethrin': 250, 125, 62.5 \\
\hline 3,4 & Contact & $\begin{array}{l}\text { Fluralaner: 3.9-62.5; spinosad: } \\
\text { 250- 4000; phoxim: } 125-2,000 ; \\
\text { propoxur, deltamethrin: } 62.5-1000\end{array}$ \\
\hline 5 & Contact & $\begin{array}{l}\text { Fluralaner: } 15.6-125 \text {; spinosad, } \\
\text { phoxim: 500-4000; propoxur, } \\
\text { deltamethrin: } 125-1000\end{array}$ \\
\hline 6 & Contact & $\begin{array}{l}\text { Fluralaner, propoxur, } \\
\text { cypermethrin: } 1.95-1000\end{array}$ \\
\hline
\end{tabular}

${ }^{\mathrm{a}}$ Due to the low number of live mites available, only deltamethrin was tested dried at room temperature for approximately $24 \mathrm{~h}$. From 60 to 400 mites in Study 1, and approximately 50 mites in Study 6, were placed onto each filter paper which was then triple-folded and sealed with a tacker, allowing direct contact between mites and the test solutions. After incubation for approximately $48 \mathrm{~h}$, in Study 1 at approximately $22{ }^{\circ} \mathrm{C}$ and $90 \%$ relative humidity (RH), and in Study 6 at $23 \pm 1{ }^{\circ} \mathrm{C}$ and $80 \pm 5 \% \mathrm{RH}$, the filter papers were opened for assessment of the mites.

In Studies 2 to 5, actives were dissolved in 1-butanol + olive oil (DAB10) to obtain a $1 \%$ stock solution (100 mg substance $/ 10 \mathrm{ml}$ solvent). Test concentrations were prepared by further 1:2 dilutions with deionized water. Filter papers $(8 \times 10 \mathrm{~cm})$ were coated with $800 \mu \mathrm{l}$ of the final test solutions or with solvent only (control), and left for drying for $48 \mathrm{~h}$ at room temperature. Impregnated papers were folded and closed with clamps on two sides. Approximately 100 mites were placed into the opening of each filter paper which was then sealed and placed in a holding apparatus (Fig. 2) for $48 \mathrm{~h}$ at 40 to $50 \% \mathrm{RH}$ and temperature of 20 to $23{ }^{\circ} \mathrm{C}$. Diluent with no miticide was used as a negative control, and an additional control was not exposed to either vehicle or active.

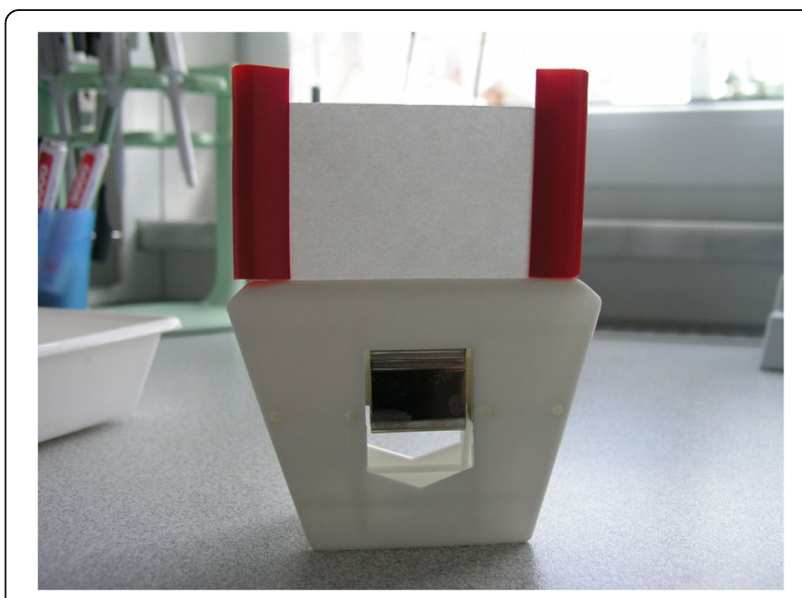

Fig. 2 Filter paper package used in the contact tests 


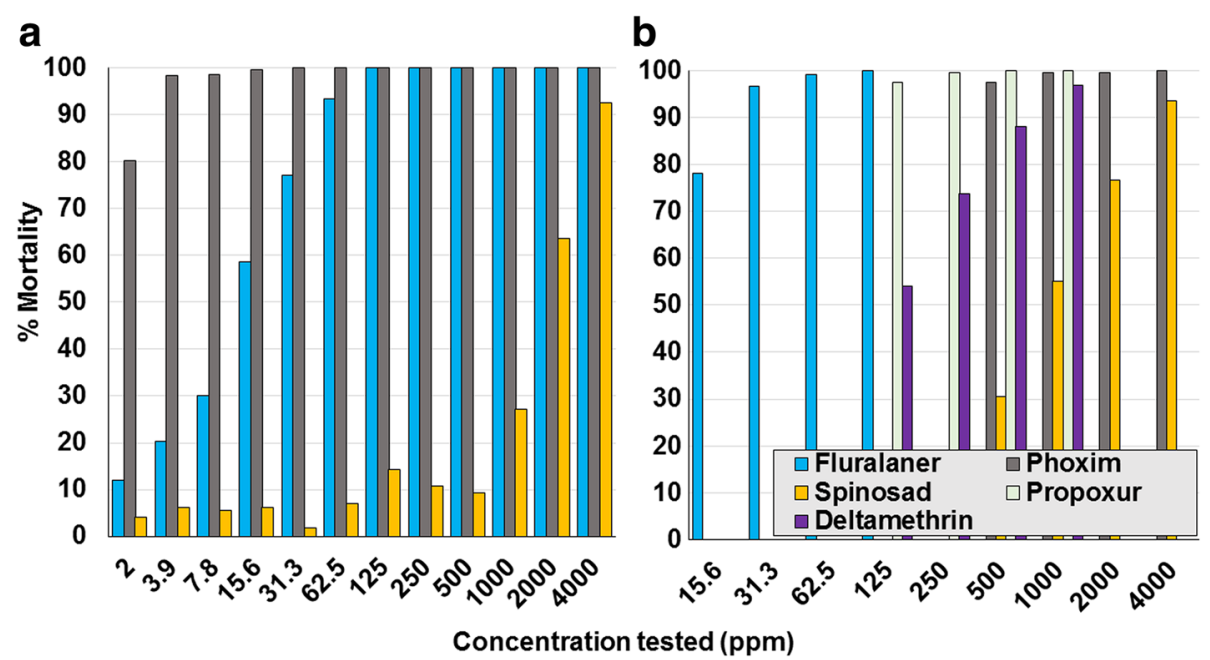

Fig. 3 Assay results from the two laboratories that tested contact sensitivity of the 2001 laboratory isolate. a Laboratory 1. b Laboratory 2

\section{Immersion contact and feeding tests (Study 1)}

For the immersion (larval sandwich) test, mites were placed between two filter papers and $5 \mathrm{ml}$ of test solution was added. Mites were immersed (between the filter papers) in fluralaner (0.031 to $4000 \mathrm{ppm}$ ), phoxim or spinosad solutions (each 2 to $4000 \mathrm{ppm}$ ). After approximately three minutes, the solution was poured off, the filter papers were dried with towelling paper and the mites were then placed onto a fresh triple folded filter paper that was sealed (as a sachet). After incubation for $48 \mathrm{~h}$, at approximately $22^{\circ} \mathrm{C}$ and $90 \% \mathrm{RH}$, all mites were counted. There were two replicates for each test compound and the two controls, one containing DMSO solvent and one untreated.

For the feeding test, between 100 and 150 mites were transferred into plastic vials (diameter $20 \mathrm{~mm}$ ), each closed with a membrane (Parafilm ${ }^{\circ}$ ) that had been treated with mite-attractant solution prepared from an ethanol-butyric acid extraction of a mixture containing minced chicken skin and feathers. The vials were then placed into a glass dish containing 2 $\mathrm{ml}$ of a warmed blood solution comprised of a mixture of defibrinized sheep blood and stock solution,

Table 2 Lethal concentration, based on 90\% mortality ( $\left(C_{90}\right)$, of tested actives against different D. gallinae isolates

\begin{tabular}{|c|c|c|c|c|c|c|}
\hline \multirow[t]{2}{*}{ Study } & \multirow[t]{2}{*}{ Isolate (year, country) } & \multicolumn{5}{|l|}{$\mathrm{LC}_{90}(\mathrm{ppm})$} \\
\hline & & Fluralaner & Pyrethroids & Phoxim & Propoxur & Spinosad \\
\hline 1 & Lab 1 (2001, Germany) & 62.5 & $\mathrm{nt}$ & $2.0-3.9$ & nt & $2000-4000$ \\
\hline 2 & Field 1 (2014, Germany) & $\mathrm{nt}$ & $>250(\mathrm{D})$ & $\mathrm{nt}$ & $\mathrm{nt}$ & $\mathrm{nt}$ \\
\hline 3 & Field 2 (2014, France) & $7.8-15.6$ & $>1000$ (D) & $>1000$ & $>1000$ & $1000-2000$ \\
\hline 4 & Field 3 (2015, Spain) & $3.9-7.81$ & $>1000$ (D) & $>2000$ & $>1000$ & $>4000$ \\
\hline \multirow[t]{10}{*}{5} & Field 4 (2015, Germany) & $<15.6$ & $\mathrm{nt}$ & $2000-4000$ & nt & $1000-2000$ \\
\hline & Field 5 (2015, France) & $<15.6$ & $\mathrm{nt}$ & $>4000$ & $\mathrm{nt}$ & $1000-2000$ \\
\hline & Field 6 (2015, France) & $15.6-31.3$ & nt & $>4000$ & nt & $1000-2000$ \\
\hline & Field 7 (2015, Germany) & $<15.6$ & $\mathrm{nt}$ & $<1000$ & nt & $1000-2000$ \\
\hline & Field 8 (2015, Spain) & $<15.6$ & $\mathrm{nt}$ & $2000-4000$ & nt & $<1000$ \\
\hline & Field 9 (2015, France) & $<15.6$ & $\mathrm{nt}$ & $2000-4000$ & $\mathrm{nt}$ & $<1000$ \\
\hline & Field 10 (2015, Germany) & $<15.6$ & nt & $1000-2000$ & nt & $1000-2000$ \\
\hline & Field 11 (2015, Germany) & $<15.6$ & $\mathrm{nt}$ & $1000-2000$ & $\mathrm{nt}$ & $1000-2000$ \\
\hline & Field 12 (2015, Germany) & $15.6-31.3$ & nt & $<1000$ & nt & $2000-4000$ \\
\hline & Lab 2 (2001, Germany) & $15.6-31.3$ & 500-1000 (D) & $<500$ & $<125$ & $2000-4000$ \\
\hline 6 & Field 13 (2016, Brazil) & 125 & $>1000(C)$ & nt & $>1000(\mathrm{C})$ & nt \\
\hline
\end{tabular}

Abbreviations: Lab 1 and Lab 2, same laboratory isolate tested in different laboratories; nt, not tested; D, deltamethrin; C, cypermethrin 
Table 3 Per cent in vitro mortality of laboratory and field isolates of Dermanyssus gallinae exposed to fluralaner

\begin{tabular}{|c|c|c|c|c|c|c|c|c|c|c|}
\hline \multirow[t]{2}{*}{ Isolate } & \multicolumn{10}{|c|}{ Concentration (ppm) } \\
\hline & 2.0 & 3.9 & 7.8 & 15.6 & 31.3 & 62.5 & 125 & 1000 & 2000 & 4000 \\
\hline Lab 1 & 1.2 & 20.4 & 30.2 & 58.6 & 77.1 & 93.3 & 100 & 100 & 100 & 100 \\
\hline Lab 2 & & & 77.9 & 96.6 & 99.2 & 100 & & & & \\
\hline Field 2 & 44.6 & 87.3 & 97.2 & 98.6 & 99.6 & & & & & \\
\hline Field 3 & 87.3 & 97.2 & 99.6 & 100 & 100 & & & & & \\
\hline Field 4 & & & 98.0 & 98.5 & 99.5 & & & & & \\
\hline Field 5 & & & 96.0 & 99.0 & 98.1 & & & & & \\
\hline Field 6 & & & 88.7 & 97.7 & 98.6 & & & & & \\
\hline Field 7 & & & 96.2 & 100 & 100 & & & & & \\
\hline Field 8 & & & 100 & 100 & 100 & & & & & \\
\hline Field 9 & & & 95.0 & 99.0 & 100 & & & & & \\
\hline Field 10 & & & & 93.6 & 99.5 & 100 & & & & \\
\hline Field 11 & & & & 93.1 & 99.6 & 99.6 & & & & \\
\hline Field 12 & & & & 82.6 & 97.1 & 98.6 & & & & \\
\hline Field 13 & & & & 27.1 & 45.8 & 86.5 & 90.6 & 96.9 & & \\
\hline
\end{tabular}

and fluralaner at concentrations ranging from $10^{-7}$ to $10^{1} \mathrm{ppm}$. Control mites were exposed to either DMSO solution or to blood only. After two hours feeding in darkness, engorged mites were collected, transferred into a glass tube containing a paper strip, closed with an air-permeable lid, and incubated for $24 \mathrm{~h}$ at approximately $22{ }^{\circ} \mathrm{C}$ and $90 \% \mathrm{RH}$. The test included two controls (one DMSO solvent and one untreated control), and was carried out in three replicates. Phoxim was not included in the feeding test because of its volatility.

\section{Assessments}

The MM, expressed as a percentage and calculated for each test concentration approximately $48 \mathrm{~h}$ after contact exposure (studies 1 to 6 ), or $24 \mathrm{~h}$ after feeding (study 1 only), was calculated as follows:

Mite mortality $=\left(\mathrm{n}_{\text {dead }} / \mathrm{n}_{\text {counted }}\right) \times 100$

where $n_{\text {dead }}$ is the arithmetic mean (of all replicas) of dead mites per test concentration; $\mathrm{n}_{\text {counted }}$ is the arithmetic mean (of all replicas) of counted mites per test concentration.

The per cent MM was calculated using the formula:

$$
\operatorname{Efficacy}(\%)=\frac{M M_{T}-M M_{C}}{100-M M_{C}} \times 100
$$

where $M M_{C}$ is the per cent $M M$ of mites exposed to the control solution and $\mathrm{MM}_{\mathrm{T}}$ is the mortality of mites exposed to the test concentration. For Studies 1 and 6, a mean of the solvent and the untreated control was used as $\mathrm{MM}_{\mathrm{C}}$.

In Study 1 the $90 \%$ lethal concentrations $\left(\mathrm{LC}_{90}\right)$ were calculated using probit analysis $\left(\mathrm{SAS}^{\circ}\right.$ release 9.2). In Studies 2 to 5 the low number of tested concentrations limited $\mathrm{LC}_{90}$ estimations to a certain range. Results therefore focus on concentrations where mortalities exceeded $90 \%$, with comparisons to $\mathrm{LC}_{90}$ values calculated in Study 1.

\section{Results}

The MM in controls ranged between 0.5-16.1\% across contact assay studies. Overall numbers of moribund mites were too few to make any notable difference to the results, and so only the counts derived from dead mites are presented. Based on $\mathrm{LC}_{90}$

Table 4 Per cent in vitro mortality of laboratory and field isolates of Dermanyssus gallinae exposed to spinosad

\begin{tabular}{|c|c|c|c|c|c|c|c|c|c|c|c|c|}
\hline \multirow[t]{2}{*}{ Isolate } & \multicolumn{12}{|c|}{ Concentration (ppm) } \\
\hline & 2.0 & 3.9 & 7.8 & 15.6 & 31.2 & 62.5 & 125 & 250 & 500 & 1000 & 2000 & 4000 \\
\hline Lab 1 & 4.1 & 6.1 & 5.5 & 6.2 & 1.8 & 7.0 & 14.2 & 10.8 & 9.2 & 27.2 & 63.7 & 92.5 \\
\hline Lab 2 & & & & & & & & & 29.9 & 54.6 & 76.4 & 93.5 \\
\hline 2 & & & & & & & & 43.2 & 55.7 & 76.5 & 92.9 & 94.5 \\
\hline 3 & & & & & & & & 24.7 & 40.5 & 61.0 & 68.9 & 72.0. \\
\hline 4 & & & & & & & & & & 85.4 & 90.3 & 94.7 \\
\hline 5 & & & & & & & & & & 84.8 & 90.3 & 96.0 \\
\hline 6 & & & & & & & & & & 80.5 & 91.0 & 97.8 \\
\hline 7 & & & & & & & & & & 89.8 & 98.4 & 99.0 \\
\hline 8 & & & & & & & & & & 99.4 & 100 & 100 \\
\hline 9 & & & & & & & & & & 97.7 & 100 & 100 \\
\hline 10 & & & & & & & & & & 74.4 & 95.0 & 100 \\
\hline 11 & & & & & & & & & & 79.1 & 89.6 & 100 \\
\hline 12 & & & & & & & & & & 39.5 & 81.5 & 100 \\
\hline
\end{tabular}


Table 5 Per cent in vitro mortality of laboratory and field isolates of Dermanyssus gallinae exposed to phoxim

\begin{tabular}{|c|c|c|c|c|c|c|c|c|c|c|c|c|}
\hline \multirow[t]{2}{*}{ Isolate } & \multicolumn{12}{|c|}{ Concentration (ppm) } \\
\hline & 2.0 & 3.9 & 7.8 & 15.6 & 31.2 & 62.5 & 125 & 250 & 500 & 1000 & 2000 & 4000 \\
\hline Lab 1 & 80.3 & 98.4 & 98.6 & 99.6 & 100 & 100 & 100 & 100 & 100 & 100 & & \\
\hline Lab 2 & & & & & & & & & 97.5 & 99.6 & 99.6 & 100 \\
\hline Field 2 & & & & & & & 0.2 & 3.6 & 5.5 & 6.9 & & \\
\hline Field 3 & & & & & & & & 14.4 & 17.3 & 7.0 & 46.8 & \\
\hline Field 4 & & & & & & & & & & 23.7 & 84.7 & 91.5 \\
\hline Field 5 & & & & & & & & & & 21.7 & 27.3 & 46.2 \\
\hline Field 6 & & & & & & & & & & 4.0 & 15.0 & 80.0 \\
\hline Field 7 & & & & & & & & & & 93.1 & 100 & 100 \\
\hline Field 8 & & & & & & & & & & 30.4 & 56.1 & 98.5 \\
\hline Field 9 & & & & & & & & & & 17.6 & 77.5 & 100 \\
\hline Field 10 & & & & & & & & & & 55.8 & 95.4 & 98.6 \\
\hline Field 11 & & & & & & & & & & 48.4 & 94.0 & 100 \\
\hline Field 12 & & & & & & & & & & 98.7 & 100 & 100 \\
\hline
\end{tabular}

values, the laboratory isolate was susceptible to fluralaner (range 15.6-62.5 ppm), spinosad (2000-4000 ppm), phoxim $(2.6$ and $<500 \mathrm{ppm})$, propoxur $(<125 \mathrm{ppm})$, and deltamethrin (500-1000 ppm) (Fig. 3a, b; Tables 2, 3, 4, 5 and 6). The two laboratories that assayed the German isolate from 2001 (laboratory isolate) returned similar contact sensitivities to fluralaner, spinosad and phoxim, indicating a consistency of results despite some differences in the testing methodologies. Field isolates were sensitive to spinosad concentrations of 2000-4000 ppm (Fig. 4). Laboratory and field isolates showed similar sensitivity to fluralaner ( $\leq 125 \mathrm{ppm})$ (Fig. 5).

For phoxim, relative to the laboratory isolate $\left(\mathrm{LC}_{90}\right.$ $2.6 \mathrm{ppm})$, there was an apparent decreased contact sensitivity of two German field isolates for which $\mathrm{LC}_{90}$ had increased to between 1000-2000 ppm (field isolates 10 and 11), and one isolate for which the $\mathrm{LC}_{90}$ lay between 2000-4000 ppm (field isolate 4) (Tables 2 and 5; Fig. 6). The phoxim $\mathrm{LC}_{90}$ for French field isolates 5 and 6 were greater than $4000 \mathrm{ppm}$, a concentration at which mortalities were just 46.2 and $80.0 \%$, respectively. For the French isolate (Field 2), $\mathrm{MM}$ at the maximum tested phoxim concentration (1000 ppm) was 6.9\%. This isolate also showed reduced sensitivity to both propoxur and deltamethrin. Field isolate 3 from Spain also demonstrated similar reduced sensitivity to phoxim, propoxur and deltamethrin. Reduced sensitivity was demonstrated by field isolate 13 from Brazil to propoxur and cypermethrin: contact exposure to $250 \mathrm{ppm}$ resulted in mortality of 15.6 and $4.1 \%$, respectively, only marginally increasing to $19.8 \%$ and $15.6 \%$ at $1,000 \mathrm{ppm}$, the highest tested exposure (Table 6).

The MM following immersion in concentrations of fluralaner and phoxim exceeded $90 \%$ at $1 \mathrm{ppm}$ and 62.5 $\mathrm{ppm}$, respectively. The peak measured MM following immersion in increasing concentrations of spinosad was $80.4 \%$ at $4000 \mathrm{ppm}$ (Fig. 7). In the feeding test, MM was $100 \%$ following exposure to conentrations of $0.1 \mathrm{ppm}$ or greater. Based on the measured value leading to $\mathrm{LC}_{50}$

Table 6 Per cent in vitro mortality of laboratory and field isolates of Dermanyssus gallinae following exposure to either propoxur or pyrethroids (deltamethrin for isolates from France and Spain, cypermethrin for Brazil isolate)

\begin{tabular}{|c|c|c|c|c|c|c|c|c|c|c|}
\hline \multirow[t]{2}{*}{ Isolate } & \multicolumn{5}{|c|}{ Propoxur concentration (ppm) } & \multicolumn{5}{|c|}{ Pyrethroid concentration (ppm) } \\
\hline & 62.5 & 125 & 250 & 500 & 1000 & 62.5 & 125 & 250 & 500 & 1000 \\
\hline Lab 2 & & 97.5 & 99.6 & 100 & 100 & & 53.5 & 73.5 & 88.1 & 96.9 \\
\hline Field 1 & & & & & & 36.9 & 47.1 & 52.1 & $\mathrm{nt}$ & $\mathrm{nt}$ \\
\hline Field 2 & 7.6 & 11.6 & 10.9 & 13.2 & 13.8 & 3.3 & 6.4 & 3.1 & 3.2 & 4.9 \\
\hline Field 3 & 7.7 & 8.1 & 6.4 & 5.2 & 12.4 & 16.3 & 11.7 & 11.0 & 13.8 & 12.9 \\
\hline Field 13 & 17.7 & 30.2 & 15.6 & 15.6 & 19.8 & 2.1 & 3.1 & 4.1 & 7.3 & 15.6 \\
\hline
\end{tabular}

Notes: Commercial rate for application of propoxur is $1000 \mathrm{ppm}$. Commercial rates for application of deltamethrin and cypermethrin are 250 and 150 ppm, respectively. For field isolate 13 , for propoxur concentrations $<62.5 \mathrm{ppm}$, mite mortality ranged from 0.0 to $12.5 \%$ at 1.95 to 31.3 ppm, respectively. For cypermethrin mortality ranged from 0.0 to $1.0 \%$ at 1.95 to $31.3 \mathrm{ppm}$, respectively 


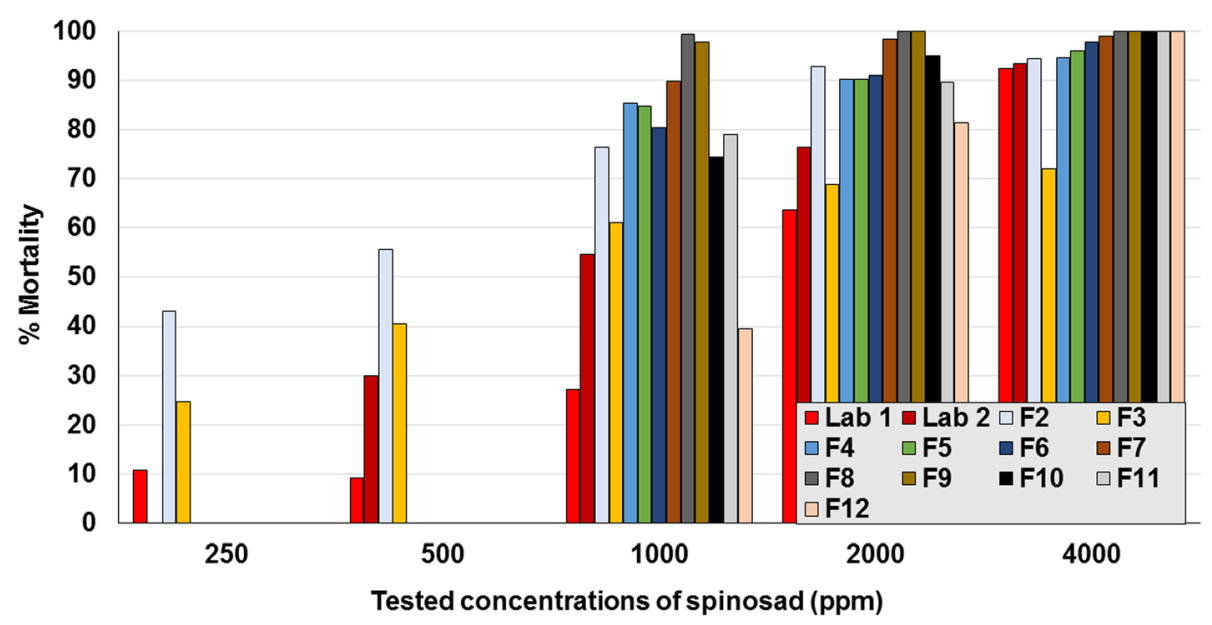

Fig. 4 Per cent mortality of Dermanyssus gallinarum following contact exposure to concentrations of spinosad (Lab 1 and Lab 2 denote the results of separate laboratories for the field strain isolated in 2001; F denotes field isolate)

and $\mathrm{LC}_{90}, \mathrm{MM}$ from exposure to fluralaner was greater in the feeding test than in either of the contact tests (filter paper and immersion) (Table 7).

\section{Discussion}

Multiple reports from individual countries have indicated that D.gallinae resistance to many acaricidal classes (organophosphates, pyrethroids, carbamates) is widespread, and is a contributing factor to the increased prevalence of poultry red mites $[4,8,22$, $24,30]$. This is the first report to describe reduced sensitivity to a range of acaricides across different countries. When compared to the commercial product label rates, the $\mathrm{LC}_{90}$ values described in this report provide further evidence that poultry red mite resistance to multiple chemical groups has developed under commercial production conditions. From the 11 field isolates originating from layer and breeder farms in France, Spain and Germany, six had a $\mathrm{LC}_{90}$ for phoxim exceeding the recommended concentration for commercial use. Deltamethrin and cypermethrin were tested on one Brazilian and three EU field isolates, and all had $\mathrm{LC}_{90}$ values exceeding the commercial product label rates of 250 and $100-$ $200 \mathrm{ppm}[31,32]$. The same was observed with propoxur for which the $\mathrm{LC}_{90}$ of the three isolates from Europe and Brazil exceeded 1000 ppm, a concentration that, in the authors experience, was used in poultry prior to its withdrawal from the European market.

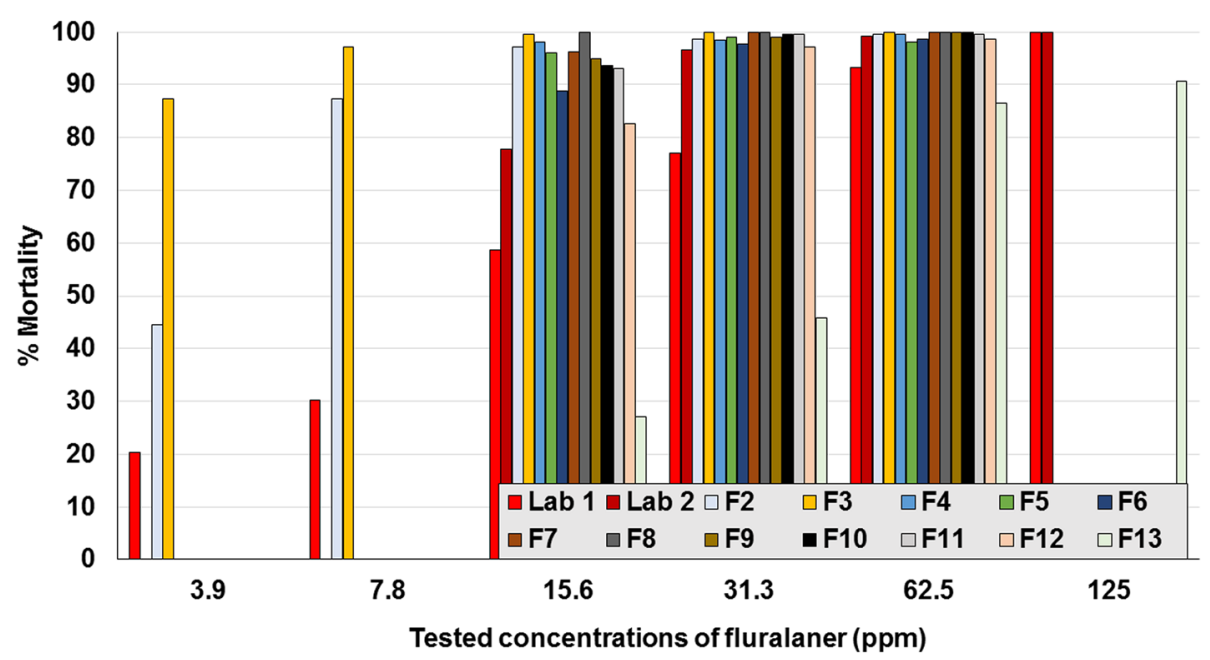

Fig. 5 Per cent mortality of Dermanyssus gallinarum following contact exposure to concentrations of fluralaner (Lab 1 and Lab 2 denote the results of separate laboratories for the field strain isolated in 2001; F denotes field isolate) 


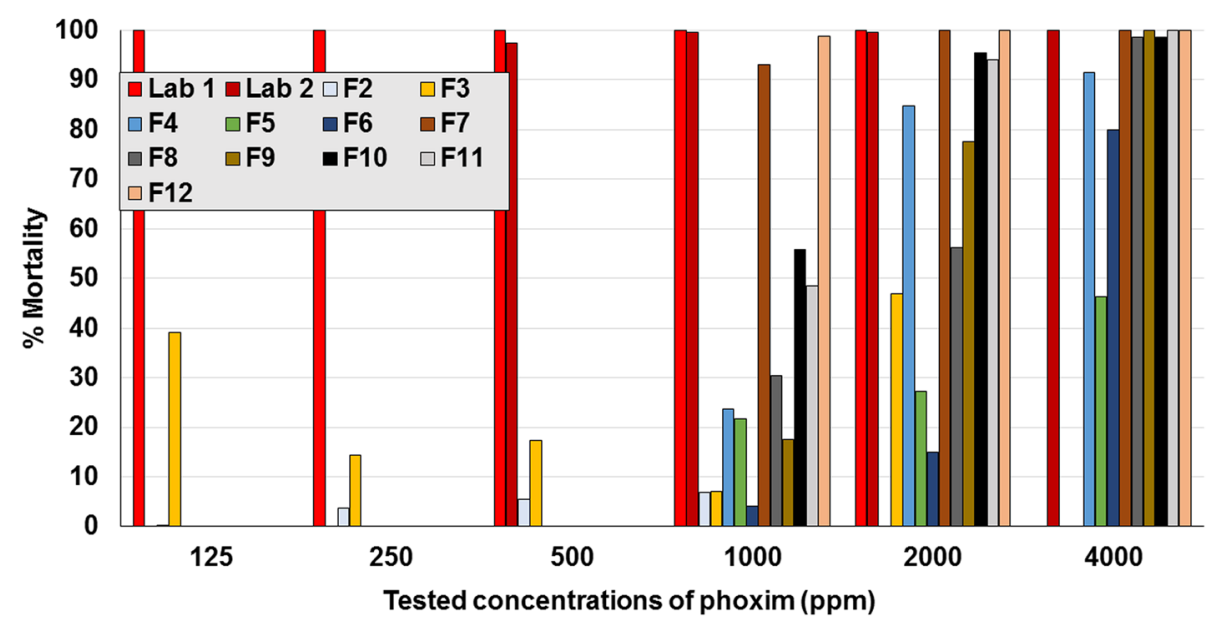

Fig. 6 Per cent mortality of Dermanyssus gallinarum following contact exposure to concentrations of phoxim (Lab 1 and Lab 2 denote the results of separate laboratories for the field strain isolated in 2001; F denotes field isolate)

In this report, three methods were used: a filter paper test corresponding to the spray application used for the traditional treatments which are contact acaricides; an immersion test; and a feeding test which is relevant to fluralaner because mites are exposed while feeding on treated birds. Irrespective of the test method, fluralaner was consistently active at low concentrations against all the tested $D$. gallinae isolates. The greatest activity of fluralaner was observed in the feeding test at extremely low lethal concentrations $\left(\mathrm{LC}_{90}<0.1 \mathrm{ppm}\right)$, approximately 1000 times lower than that obtained during the filter paper contact test. The latter test thus provides a "worst case scenario" with reference to fluralaner's systemic mode of action, especially when compared to results of other acaricides with a contact mode of action. The value of more potent systemic than contact activity for fluralaner is that the innovative mode of administration (in drinking water) enables the use of a lower and more precise amount of active, when compared with a spray application.

The spinosad $\mathrm{LC}_{90}$ of up to $4000 \mathrm{ppm}$ for the laboratory and EU field isolates with MM assessed after 48 hours, indicates that this chemical is less potent than the other tested acaricides, including fluralaner, and is consistent with previous sensitivity reports [33]. These concentrations are close to, or even exceed the spinosad recommended dose rate. The killing activity of spinosad has been reported to increase beyond 48 hours after exposure, although even after five days a concentration of 2000 ppm failed to achieve a $90 \%$ mortality of adult mites [33].

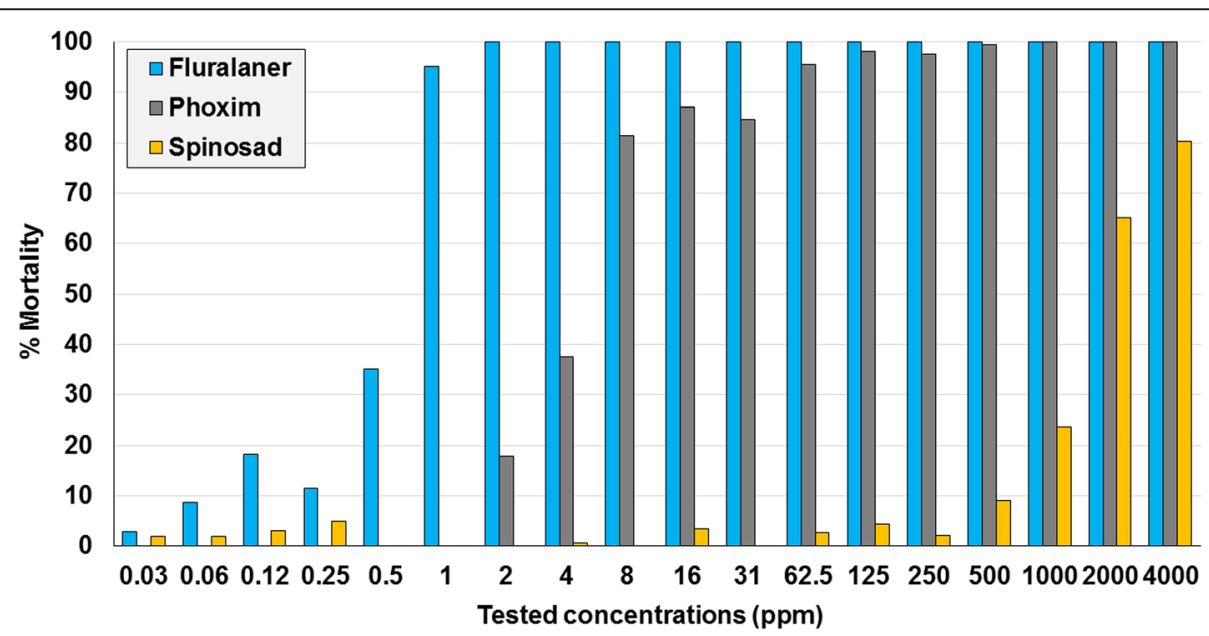

Fig. 7 Per cent mortality of the laboratory isolate of Dermanyssus gallinarum following 3-minute immersions in concentrations of fluralaner, phoxim and spinosad 
Table 7 Calculated 50\% ( $\left.\mathrm{LC}_{50}\right)$ and 90\% ( $\left(\mathrm{C}_{90}\right)$ values (ppm) of a laboratory strain of D. gallinae exposed in vitro to fluralaner, phoxim and spinosad

\begin{tabular}{|c|c|c|c|}
\hline Test & Compound & $\mathrm{LC}_{50}$ & $\mathrm{LC}_{90}$ \\
\hline \multirow[t]{3}{*}{ Filter paper contact } & Fluralaner & 11.62 & 49.93 \\
\hline & Phoxim & 0.72 & 2.63 \\
\hline & Spinosad & 1524 & $c .4000^{\mathrm{a}}$ \\
\hline \multirow[t]{3}{*}{ Immersion } & Fluralaner & 0.52 & 1.57 \\
\hline & Phoxim & 4.25 & 30.44 \\
\hline & Spinosad & 2311 & $>4000^{\mathrm{a}}$ \\
\hline Feeding & Fluralaner & 0.0125 & $<0.1^{\mathrm{a}}$ \\
\hline
\end{tabular}

${ }^{a}$ These values could not be estimated using the mathematical model

The present studies assessed MM following exposure of a 2001 isolate, and isolates from 2014 to 2015, to phoxim, propoxur and spinosad. Using the same method at the same laboratory, the results show a declining sensitivity of field isolates to phoxim and propoxur. No change was observed for spinosad which was only launched in 2012. This supports the belief that resistance to older compounds has continued to build during the last decade [8].

Results from the studies described in this report show that fluralaner is active against poultry red mites, isolated from commercial operations, showing decreased susceptibility to the most commonly used acaricides. These findings are consistent with reports of fluralaner efficacy against Ornithonyssus sylviarum, the northern fowl mite, particularly prevalent in North and Latin America, at similar exposure rates as those to which $D$. gallinae is sensitive [34]. They also align with previous results showing that fluralaner has been shown to overcome resistance in fleas, ticks or sea lice against phenylpyrazoles, amidines, organophosphates, cyclodienes, macrocyclic lactones, phenylpyrazoles, benzophenyl ureas, pyrethroids and carbamates [35].

\section{Conclusions}

The studies reported in this paper demonstrate that the poultry red mite D. gallinae is highly susceptible to the novel poultry acaricide fluralaner, with much greater sensitivity shown by exposure through feeding on blood than by contact or immersion. This differential sensitivity toward exposure through feeding aligns with fluralaner's oral administration for systemic activity against mites. Similar susceptibility to fluralaner was observed for European and Brazilian field isolates showing apparent resistance to phoxim, deltamethrin, cypermethrin and propoxur. The different mode of action than that of other poultry acaricides, and the efficacy demonstrated against isolates largely refractory to those acaricides, makes fluralaner a promising option for the control of D. gallinae, helping to reduce or avoid the costly effects of this damaging parasite.

\section{Abbreviations}

DDT: Dichlorodiphenylytrichloroethane; FAO: Food and Agriculture

Organisation; LC 90 : Lethal concentration that causes 90\% mortality; MM: Mite mortality; Ppm: Parts per million; RH: Relative humidity

\section{Acknowledgements}

The authors would like to thank Dr Bill Ryan of Ryan Mitchell Associates LLC, Maria D. Brauneis and Christina Wengenmayer of MSD and Stephen Brown of Clinglobal for guidance and support with preparation of the manuscript.

Funding

All studies were funded by MSD as part of their ongoing research programmes.

\section{Availability of data and materials}

The data supporting the conclusions of this article are included within the article. The individual study reports for in vitro studies 1 to 6 are archived by MSD.

\section{Authors' contributions}

ET organised the studies. HZ (at MSD), GL (at Zecklab) and LFAA (UNIOESTE) conducted the in vitro studies. LV and RMC supervised the study in Brazil. ASF was involved in the study design and the interpretation of the results. All authors read and approved the final manuscript.

Ethics approval and consent to participate

As these were in vitro studies with no host animals involved, neither ethics approval nor informed consent were required.

Consent for publication

Not applicable.

\section{Competing interests}

MSD performed and funded study 1, and funded studies 2 to 6 .

\section{Publisher's Note}

Springer Nature remains neutral with regard to jurisdictional claims in published maps and institutional affiliations.

\section{Author details}

'MSD Animal Health Innovation GmbH, Zur Propstei, 55270 Schwabenheim, Germany. ${ }^{2}$ ZeckLab, Up'n Kampe 3, 30938 Burgwedel, Germany. ${ }^{3}$ State University of Western Paraná (UNIOESTE), Cascavel, PR, Brazil. ${ }^{4}$ MSD Animal Health, São Paulo, Brazil. ${ }^{5}$ Merck Animal Health, 2 Giralda Farms, Madison, NJ 07940, USA.

Received: 5 April 2018 Accepted: 15 June 2018

Published online: 25 June 2018

References

1. Pritchard J, Kuster T, Sparagano O, Tomley F. Understanding the biology and control of the poultry red mite Dermanyssus gallinae: a review. Avian Pathol. 2015:44:143-53.

2. Harrington DWJ, George DR, Guy JH, Sparagano OAE. Opportunities for integrated pest management to control the poultry red mite, Dermanyssus gallinae. Worlds Poult Sci J. 2011;67:83-94.

3. Zdybel J, Karamon J, Cencek T. In vitro effectiveness of selected acaricides against red poultry mites (Dermanyssus gallinae De Geer, 1778) isolated from laying hen battery cage farms localised in different regions of Poland. Bull Vet Inst Pulawy. 2011;55:411-6.

4. Flochlay Sigognault A, Thomas E, Sparagano O. Poultry red mite (Dermanyssus gallinae) infestation: a broad impact parasitological disease that still remains a significant challenge for the egg-laying industry in Europe. Parasit Vectors. 2017;10:357.

5. Sparagano OAE, George DR, Harrington DWJ, Giangaspero A. Significance and control of the poultry red mite, Dermanyssus gallinae. Annu Rev Entomol. 2014:59:447-66.

6. Chauve C. The poultry red mite Dermanyssus gallinae (De Geer, 1778): current situation and future prospects for control. Vet Parasitol. 1998;79: 239-45. 
7. Kilpinen O, Roepstorff A, Permin A, Nørgaard-Nielsen G, Lawson LG, Simonsen HB. Influence of Dermanyssus gallinae and Ascaridia galli infections on behaviour and health of laying hens (Gallus gallus domesticus). Br Poult Sci. 2005:46:26-34.

8. Abbas R, Colwell D, labal Z, Khan A. Acaricidal drug resistance in poultry red mite (Dermanyssus gallinae) and approaches to its management. Worlds Poult Sci J. 2014;70:113-24.

9. George DR, Finn RD, Graham KM, Mul MF, Maurer V, Moro CV, et al. Should the poultry red mite Dermanyssus gallinae be of wider concern for veterinary and medical science? Parasit Vectors. 2015;8:178.

10. Cafiero MA, Camarda A, Circella E, Santagada G, Schino G, Lomuto M. Pseudoscabies caused by Dermanyssus gallinae in Italian city dwellers: a new setting for an old dermatitis. J Eur Acad Dermatol Venereol. 2008;22: 1382-3.

11. Cafiero MA, Galante D, Camarda A, Giangaspero A, Sparagano O. Why dermanyssosis should be listed as an occupational hazard. Occup Environ Med. 2011;68:628.

12. Sparagano O, Pavlićević A, Murano T, Camarda A, Sahibi H, Kilpinen O, et al. Prevalence and key figures for the poultry red mite Dermanyssus gallinae infections in poultry farm systems. Exp Appl Acarol. 2009;48:3-10.

13. Kirkwood AC. Some observations on the feeding habits of the poultry mites Dermanyssus gallinae and Liponyssus sylviarum. Entomol Exp Appl. 1968;11: 315-20.

14. Liebisch A, Liebisch G. Biologie, Schäden und Bekämpfung beim Befall durch die Rote Vogelmilbe (Dermanyssus gallinae). Lohmann Inf. 2003;4:1-7.

15. Maurer $\vee$, Baumgärtner J. Temperature influence on life table statistics of the chicken mite Dermanyssus gallinae (Acari: Dermanyssidae). Exp Appl Acarol. 1992;15:27-40

16. Nordenfors $\mathrm{H}$, Höglund J, Uggla A. Effects of temperature and humidity on oviposition, molting, and longevity of Dermanyssus gallinae (Acari: Dermanyssidae). J Med Entomol. 1999;36:68-72.

17. Kirkwood AC. Longevity of the mites Dermanyssus gallinae and Liponyssus sylviarum. Exp Parasitol. 1963;14:358-66.

18. Marangi M, Morelli V, Pati S, Camarda A, Cafiero MA, Giangaspero A Acaricide residues in laying hens naturally infested by red mite Dermanyssus gallinae. PLoS One. 2012:7:3-8.

19. Brockotter F. Belgian-Dutch fipronil scandal expands. http://www. poultryworld.net/Home/General/2017/7/Belgian-Dutch-Fipronil-scandalexpands-164304E/. Accessed 27 Dec 2017.

20. Thind BB, Ford HL. Assessment of susceptibility of the poultry red mite Dermanyssus gallinae (Acari: Dermanyssidae) to some acaricides using an adapted filter paper based bioassay. Vet Parasitol. 2007;144:344-8.

21. Zeman P. Encounter the poultry red mite resistance to acaricides in Czechoslovak poultry-farming. Folia Parasitol (Praha). 1987;34:369-73.

22. Beugnet F, Chauve C, Gauthey M, Beert L. Resistance of the red poultry mite to pyrethroids in France. Vet Rec. 1997;140:577-9.

23. Marangi M, Cafiero MA, Capelli G, Camarda A, Sparagano OAE, Giangaspero A. Evaluation of the poultry red mite, Dermanyssus gallinae (Acari: Dermanyssidae) susceptibility to some acaricides in field populations from Italy. Exp Appl Acarol. 2009:48:11-8.

24. Pavlicevic A, Pavlovic I, Stajkovic N, Pešic B. Evidence for resistance to carbaryl in poultry red mites from the Republic of Serbia and Montenegro. Scientific Papers: Animal Science and Biotechnologies. 2016:49:222-5.

25. European Medicines Agency. EMA Marketing Authorisation Exzolt. 2017. http://www.ema.europa.eu/docs/en_GB/document_library/EPAR_-_Public_ assessment_report/veterinary/004344/WC500236953.pdf. Accessed 12 June 2018.

26. Brauneis $M$, Williams $H$, Zoller $H$, Zschiesche $E$, Heckeroth $A$. The acaricidal speed of kill of orally administered fluralaner against poultry red mites (Dermanyssus gallinae) on laying hens and its impact on mite reproduction. Parasit Vectors. 2017;10:594

27. Thomas E, Chiquet M, Sander B, Zschiesche E, Sigognault-Flochlay A. Field efficacy and safety of fluralaner solution for administration in drinking water for the treatment of poultry red mites (Dermanyssus gallinae) infestations in commercial flocks in Europe. Parasit Vectors. 2017;10:457.

28. Gassel M, Wolf C, Noack S, Williams H, Il T. The novel isoxazoline ectoparasiticide fluralaner: selective inhibition of arthropod $\mathrm{Y}$-aminobutyric acid- and L-glutamate-gated chloride channels and insecticidal/acaricidal activity. Insect Biochem Mol Biol. 2014;45:111-24.

29. FAO Working Group on Parasite Resistance: Resistance management and integrated parasite control in ruminants: Guidelines. Module 1. Ticks:
Acaricide Resistance: Diagnosis, Management and Prevention. Publisher location: FAO; 2004, p. 25-77 http://www.fao.org/tempref/docrep/fao/010/ ag014e/ag014e05.pdf. Accessed 12 June 2018.

30. Abdel-Ghaffar F, Semmler M, Al-Rasheid K, Mehlhorn H. In vitro efficacy of ByeMite and Mite-Stop on developmental stages of the red chicken mite Dermanyssus gallinae. Parasitol Res. 2009;105:1469-71.

31. Rambosal K. (2.5\% deltamethrin). Sicherheitsdatenblatt gemäß Verordnung. http://www.hagra.de/sicherheitsdatenblaetter/sdb/sdbs/607/4814.pdf. Accessed 16 Feb 2018.

32. Ectic (Cypermethrin; Tortuga). http://mercado.ruralcentro.com.br/produtos/ 12665/ectic-pulverizacao-tortuga. Accessed 16 Feb 2018.

33. Elector Technical Manual, 2012 https://www.salmonella360.com/cms3/ assets/fullsize/1045. Accessed 16 Feb 2018.

34. Mullens BA, Murillo AC, Zoller H, Heckeroth AR, Jirjis F, Flochlay-Sigognault A. Comparative in vitro evaluation of contact activity of fluralaner, spinosad, phoxim, propoxur, permethrin and deltamethrin against the northern fowl mite, Ornithonyssus sylviarum. Parasit Vectors. 2017;10:358.

35. Committee for Medicinal Products for Veterinary Use (CVMP). CVMP Assessment Report for Bravecto (EMEAN/C/002526/0000). 2013. http://www.ema.europa.eu/ docs/en_GB/document_library/EPAR_-_Public_assessment_report/veterinary/ 002526/WC500163860.pdf. Accessed 23 Feb 2018.

\section{Ready to submit your research? Choose BMC and benefit from:}

- fast, convenient online submission

- thorough peer review by experienced researchers in your field

- rapid publication on acceptance

- support for research data, including large and complex data types

- gold Open Access which fosters wider collaboration and increased citations

- maximum visibility for your research: over $100 \mathrm{M}$ website views per year

At BMC, research is always in progress.

Learn more biomedcentral.com/submissions 